\title{
Correction: Assessing Public Interest Based on Wikipedia's Most Visited Medical Articles During the SARS-CoV-2 Outbreak: Search Trends Analysis
}

Jędrzej Chrzanowski $^{1^{*}}$; Julia Sołek ${ }^{1,2^{*}}$, MD; Wojciech Fendler ${ }^{1}, \mathrm{MD}, \mathrm{PhD}$; Dariusz Jemielniak ${ }^{3}$, PhD

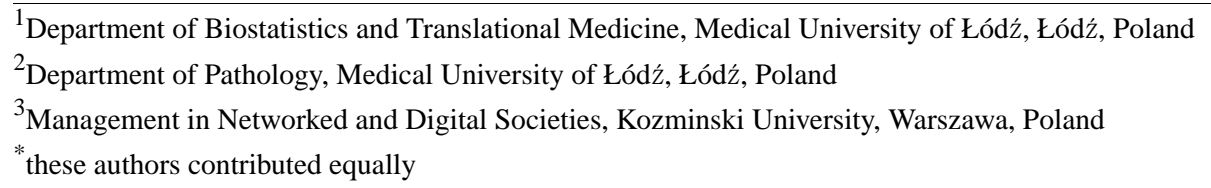

\section{Corresponding Author:}

Wojciech Fendler, MD, PhD

Department of Biostatistics and Translational Medicine

Medical University of Łódź

Mazowiecka 15

Łódź, 92-215

Poland

Phone: 48422722585

Email: wojciech.fendler@umed.lodz.pl

\section{Related Article:}

Correction of: https://jmir.org/2021/4/e26331

(J Med Internet Res 2021;23(4):e29598) doi: 10.2196/29598

In "Assessing Public Interest Based on Wikipedia's Most Visited Medical Articles During the SARS-CoV-2 Outbreak: Search Trends Analysis" (J Med Internet Res 2021;23(4):e26331) the authors noted two errors.

Due to a system error, the name of one author, Wojciech Fendler, was replaced with the name of another author on the paper, Dariusz Jemielniak. In the originally published paper, the order of authors was listed as follows:

Jędrzej Chrzanowski; Julia Sołek; Dariusz Jemielniak; Dariusz Jemielniak

This has been corrected to:

Jędrzej Chrzanowski; Julia Sołek; Wojciech Fendler; Dariusz Jemielniak
In the originally published paper, the ORCID of author 'Wojciech Fendler' was incorrectly published as follows:

Wojciech Fendler: 0000-0002-3745-7931

This has been corrected to:

Wojciech Fendler: 0000-0002-5083-9168

The correction will appear in the online version of the paper on the JMIR Publications website on April 15, 2021, together with the publication of this correction notice. Because this was made after submission to PubMed, PubMed Central, and other full-text repositories, the corrected article has also been resubmitted to those repositories.

This is a non-peer-reviewed article. Submitted 13.04.21; accepted 13.04.21; published 15.04.21.

Please cite as:

Chrzanowski J, Sotek J, Fendler W, Jemielniak D

Correction: Assessing Public Interest Based on Wikipedia's Most Visited Medical Articles During the SARS-CoV-2 Outbreak: Search Trends Analysis

J Med Internet Res 2021;23(4):e29598

URL: https://www.jmir.org/2021/4/e29598

doi: $10.2196 / 29598$

PMID: 
CJędrzej Chrzanowski, Julia Sołek, Wojciech Fendler, Dariusz Jemielniak. Originally published in the Journal of Medical Internet Research (http://www.jmir.org), 15.04.2021. This is an open-access article distributed under the terms of the Creative Commons Attribution License (https://creativecommons.org/licenses/by/4.0/), which permits unrestricted use, distribution, and reproduction in any medium, provided the original work, first published in the Journal of Medical Internet Research, is properly cited. The complete bibliographic information, a link to the original publication on http://www.jmir.org/, as well as this copyright and license information must be included. 